\title{
Pre-inflationary genesis with CMB B-mode polarization
}

\author{
Zhi-Guo Liu ${ }^{1 *}$, Hong $\mathrm{Li}^{2,3 \dagger}$, and Yun-Song Piao ${ }^{1,4 \ddagger}$ \\ ${ }^{1}$ School of Physics, University of Chinese Academy of Sciences, Beijing 100049, P.R.China \\ 2 Key Laboratory of Particle Astrophysics, \\ Institute of High Energy Physics, Chinese Academy of Science, \\ P.O.Box 918-4, Beijing 100049, P.R.China \\ 3 National Astronomical Observatories, \\ Chinese Academy of Sciences, Beijing 100012, P.R.China and \\ 4 State Key Laboratory of Theoretical Physics, Institute of Theoretical Physics, \\ Chinese Academy of Sciences, P.O. Box 2735, Beijing 100190, China
}

\begin{abstract}
Recent B-mode polarization observation seems to imply the tensor tilt $n_{T} \gtrsim 1$ at large angular scale, if the primordial signal is dominated. We show that for a primordial universe, which is in a slowly expanding genesis phase before the slow-roll inflation, the primordial tensor spectrum will get a large-scale cutoff, i.e. $n_{T} \gtrsim 1$ at large scales while $n_{T} \simeq 0$ at small scale. We find that this inflationary scenario not only may be consistent with the observation, but also predicts a large-scale anomaly in BB power spectrum, i.e. due to the large suppression of tensor perturbation amplitude we will hardly see the reionization bump at low-l, which may be falsified by the Planck polarization data.
\end{abstract}

PACS numbers:

\footnotetext{
* Email: liuzhiguo08@mails.ucas.ac.cn

$\dagger$ Email: hongli@ihep.ac.cn

$\ddagger$ Email: yspiao@ucas.ac.cn
} 
The discovery of the cosmic microwave background (CMB) B-mode polarization, contributed by the primordial gravitational wave, is crucial for understanding the physics of the early universe, especially solidifying our confidence that inflation has ever occurred. Recently, the BICEP2 collaboration has declared the detection of the CMB B-mode polarization [1]. However, it might be wholly or partly due to polarized dust emission [2], see also [3].

Though the primordial B-mode polarization could be partially caused by other sources [4], [5], it is highly-probably induced by the primordial tensor perturbation. The Planck temperature data have put the constraint $r<0.11$ (95\% C.L.) [6] on the tensor amplitude, but no limit for the tensor tilt $n_{T}$. Recent B-mode polarization data, after the dust is neglected, seem to favor $n_{T} \gtrsim 1$ e.g.|7], [8],[10],[11],[12],[13], see also [9] for the analysis for the consistence of slow-roll inflationary models. However, despite this result, it is interesting to think over the physics of inflationary universe relevant with $n_{T} \gtrsim 1$.

The conventional inflation model requires $n_{T}=-2 \epsilon<0$, in which $\epsilon=-\dot{H} / H^{2} . n_{T}>0$ implies either new mechanism or non-Bunch-Davis vacuum is involved during inflation, e.g. [14], [15], [16], or the NEC is broken, see e.g. [17] for comments. In past, inflationary model with the NEC violation has been also proposed [18],[19]. However, since the almost scaleinvariance of the scalar perturbation requires $|\epsilon| \ll 1$, we generally have $\left|n_{T}\right| \ll 1$ during the inflation. Thus $n_{T} \gtrsim 1$ seems be a challenge for the inflationary scenario. There is an alternative to inflation, in which the primordial universe is slowly expanding, i.e. genesis scenario [20], 21], [22], also earlier [23], see [24] for a review. In this scenario, $n_{T}=2$, thus its tensor amplitude is exponentially low at large scale, which is negligible. The case is similar to that in ekpyrotic scenario [25].

However, if $n_{T} \gtrsim 1$ is only at large angular scale, while at intermediate and small scales $n_{T} \simeq 0$ is satisfied, it will be possible to put $r$ at large and intermediate scales in a detectable level. In this case, $n_{T} \gtrsim 1$ at large angular scale might be a potential reflection of the preinflationary evolution.

The pre-inflationary evolution may bring signals in TT power spectrum at large angular scale, e.g. [26], [27], [28], which mainly imprinted by the primordial scalar perturbation. Besides the evolution of $a$, the scalar spectrum is also affected by the model parameters associated with the field dynamics. However, the tensor perturbation spectrum only encodes the behavior of $a$, which straightly records the evolution of the primordial universe. Thus 
$n_{T} \gtrsim 1$ at large scale will be a significant hint of what occurring before inflation. The idea of a short period of inflation has been studied in Ref.[29], without a pre-inflationary NEC violation. However, a pre-inflationary evolution with NEC violation is interesting, since it helps to solve the initial singularity problem of inflationary universe [30].

Here, we will propose an inflationary scenario, in which the pre-inflationary evolution of the primordial universe is a slowly expanding genesis phase. We find that this preinflationary genesis will make the primordial tensor spectrum get a large-scale cutoff, i.e. $n_{T}>1$ at large scale, while $n_{T} \simeq 0$ at small scale. Thus this scenario not only may be consistent with the observation, but also predicts a large-scale anomaly in BB power spectrum, i.e. due to the large suppression of tensor perturbation amplitude we will hardly see the reionization bump at $l<10$, which may be falsified by the upcoming Planck polarization data.

We begin with briefly illustrating the background evolution and the scalar perturbation. Before the slow-roll inflation, the universe is in a slowly expanding genesis phase,

$$
a \sim e^{\frac{1}{M^{b}\left(t_{*}-t\right)^{b}}}
$$

where $b>0$ and $t$ runs from negative infinite. Initially $|t| \gg\left|t_{*}\right|$ and $M^{b}\left(t_{*}-t\right)^{b} \gg 1$, the universe is slowly expanding [20],[21],[22], see [31],[32] for alternatives. Here,

$$
H \sim \frac{1}{M^{b}\left(t_{*}-t\right)^{b+1}},
$$

which initially may be negligible, but rapidly increases with the time. Thus $\epsilon=-\dot{H} / H^{2} \ll$ -1 breaks NEC, which seems implying the ghost instability. However, the ghost instability may be dispelled by applying Galileon field [33]. We may find initially $|\epsilon| \sim M^{b}\left(t_{*}-t\right)^{b} \gg 1$ and rapidly decreases with time. When $|\epsilon|<1$, the slow expansion of universe ends, after this

$$
H=H_{*} \sim \frac{1}{\left|t_{*}\right|} \simeq H_{\text {inf }}
$$

is unchanged and the slow-roll inflation will begin, in which

$$
a \sim e^{H_{i n f}\left(t-t_{*}\right)}
$$

and $\epsilon_{\text {inf }} \ll 1$. We plot Fig 1 for illustrating this scenario and the evolution of primordial perturbations. 


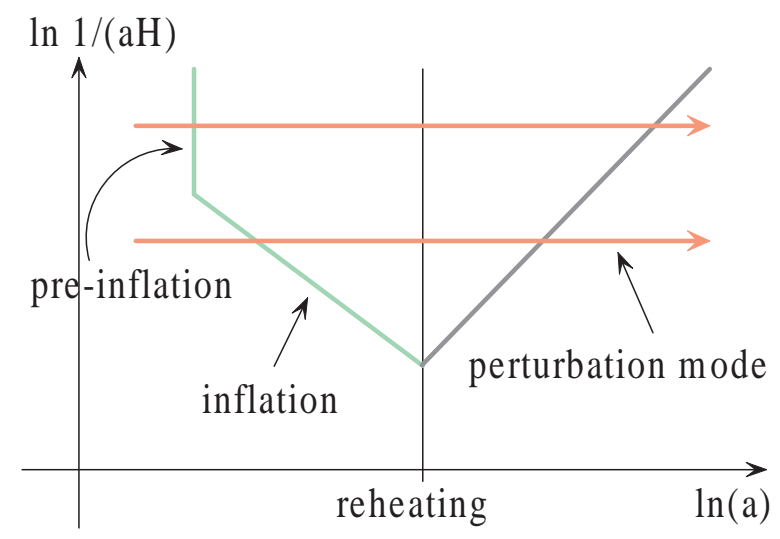

FIG. 1: The sketch of the evolution of primordial perturbations in our scenario. The perturbation mode at large scale is from the pre-inflationary genesis phase, while others are from the inflationary phase.

In slow-roll inflationary phase, the scalar perturbation is scale-invariant, i.e. $n_{\mathcal{R}}-1 \ll$ 1. In pre-inflationary genesis phase, the scalar perturbation has been investigated. In Refs. [21], [34], $a \sim e^{\frac{1}{\left(t_{*}-t\right)^{4}}}$, which gives the scale-invariant adiabatic perturbation,

$$
\mathcal{P}_{\mathcal{R}}=\frac{H_{*}^{2}}{8 \pi^{2} \epsilon_{*} M_{P}^{2}}
$$

with $H_{*}$ defined in (33). In Ref.[20], $a \sim e^{\frac{1}{\left(t_{*}-t\right)^{2}}}$, the adiabatic perturbation is not scaleinvariant, but the perturbations of conformal light scalar fields may be scale invariant [35],[20], [36], which will contribute the curbature perturbation. Thus the pre-inflationary scalar spectrum may be naturally coincident with that of slow-roll inflation, in spite of the tensor perturbation. Here, since BB is contributed only by the tensor perturbation, we will not involve the scalar perturbation, and only require that it is consistent with all data. We will clarify the details of the model building elsewhere.

However, the pre-inflationary tensor spectrum can hardly be the same with that of slowroll inflation. We will investigate the primordial tensor perturbation in this scenario. The action of the tensor perturbation $h_{i j}$ is

$$
S_{2} \sim \int d \eta d^{3} x \frac{a^{2} M_{P}^{2}}{4}\left(h_{i j}{ }^{2}-\left(\partial h_{i j}\right)^{2}\right)
$$

The equation of $h_{i j}$ in momentum space is

$$
v_{k}^{\prime \prime}+\left(k^{2}-\frac{a^{\prime \prime}}{a}\right) v_{k}=0,
$$


after $v_{k} \equiv a M_{P} h_{i j, k} / \sqrt{2}$ is defined, where' is the derivative with respect to conformal time $\eta=\int d t / a$.

We, for simplicity, will adopt an instantaneous matching of backgrounds (11) and (41) at $\eta=0$, and have

$$
\begin{gathered}
a \simeq a_{*}, \text { for preinflationary genesis phase, } \\
\frac{a_{*}}{1-\mathcal{H}_{*} \eta}, \text { for inflationary phase }
\end{gathered}
$$

respectively, $\mathcal{H}_{*}$ is the comoving $H_{*}$ at $\eta=0$, which sets the slow-roll inflationary scale by $H_{\text {inf }}=H_{*}=\mathcal{H}_{*} / a_{*}$.

When $k^{2} \gg \frac{a^{\prime \prime}}{a}$, i.e. the perturbation is deeply inside its horizon, $v_{k}$ oscillates with a constant amplitude,

$$
v_{k} \sim \frac{1}{\sqrt{2 k}} e^{-i k \eta}
$$

When $k^{2} \ll \frac{a^{\prime \prime}}{a}$, i.e. the perturbation is far outside the horizon, in the genesis phase the solution of Eq.(7) is

$$
v_{k}=\sqrt{\frac{\pi}{4} x} H_{1 / 2}^{(1)}(x),
$$

where $x=k / \mathcal{H}_{*}-k \eta, H_{1 / 2}^{(1)}$ is the $1 / 2$ th order Hankel function of the first kind, while in the inflationary phase the solution of Eq.(7) is

$$
v_{k}=\sqrt{x} c_{1} H_{3 / 2}^{(1)}(x)+\sqrt{x} c_{2} H_{3 / 2}^{(2)}(x),
$$

where $H_{3 / 2}^{(1)}$ and $H_{3 / 2}^{(2)}$ are the $3 / 2$ th-order Hankel function of the first kind and the second kind, respectively, $c_{1}$ and $c_{2}$ are only dependent on $k$.

The continuity of $h_{i j}$ gives $c_{1}$ and $c_{2}$. Thus the spectrum of $h_{i j}$ is $\mathcal{P}_{T}=\frac{2 k^{3}}{\pi^{2}}\left|\frac{v_{k}}{a}\right|^{2}$, which equals to

$$
\begin{aligned}
\mathcal{P}_{T} & =\frac{4 H_{\text {inf }}^{2}}{\pi^{3} M_{P}^{2}} k\left|c_{1}-c_{2}\right|^{2} \\
& =\mathcal{P}_{T, \text { inf }}\left(1+\frac{\cos 2 \tilde{k}}{\tilde{k}^{2}}-\frac{\sin 2 \tilde{k}}{\tilde{k}^{3}}+\frac{\sin ^{2} \tilde{k}}{\tilde{k}^{4}}\right),
\end{aligned}
$$

where $\mathcal{P}_{T, \text { inf }}=\frac{2 H_{\text {inf }}^{2}}{\pi^{2} M_{P}^{2}}$ is that of the slow-roll inflation, and $\tilde{k}=k / \mathcal{H}_{*}$ is defined for simplicity. When $\tilde{k} \ll 1$, i.e. $k \ll \mathcal{H}_{*}$, we can have approximately

$$
\mathcal{P}_{T} \sim \tilde{k}^{2}
$$




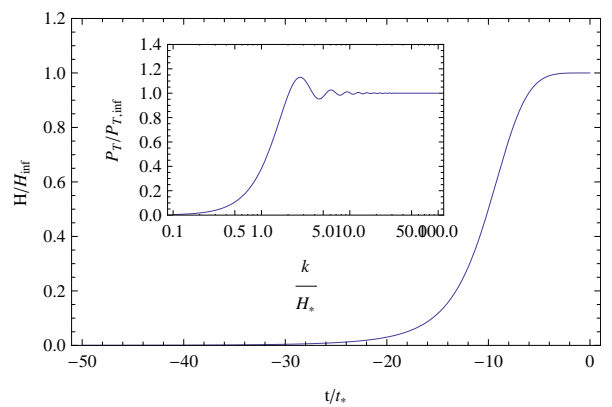

FIG. 2: The sketch of the evolution of $H$. During the pre-inflationary phase, it initially is negligible, but rapidly increases with the time. After $H=H_{\text {inf }}$, it becomes nearly unchanged, the slow-roll inflation will begin. The inset is the power spectrum (12) of the primordial tensor perturbation in our scenario with respect to $k / \mathcal{H}_{*}$, which has a large-scale cutoff, i.e. $n_{T} \gtrsim 1$ for $k<\mathcal{H}_{*}$ and $n_{T} \simeq 0$ for $k>\mathcal{H}_{*}$. The figure of the evolution of $H$ with the time should be almost coincident with that of the tensor spectrum with respect to $k$, because the amplitude of tensor mode with wavelength $1 / k$ is $\sim H$ at the time $k=a H$.

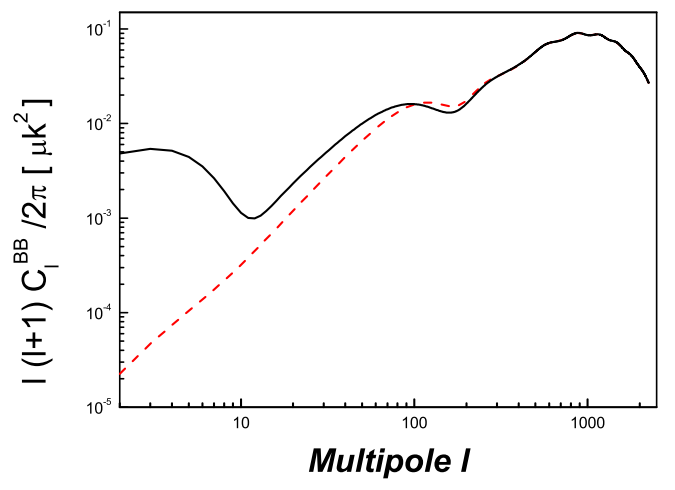

FIG. 3: Theoretical CMB BB power spectra for the conventional slow-roll inflationary scenario (black solid line), in which the tensor spectrum is power-law, and $\Omega_{b} h^{2}=0.02184, \Omega_{c} h^{2}=0.118$, $\tau=0.08,100 \Theta_{s}=1.04, n_{\mathcal{R}}=0.96 A_{s}=2.14 \times 10^{-9}$, and our scenario (red dashed line), in which $n_{T}$ is replaced with Eq.(12).

where all terms with $1 / \tilde{k}^{2}$, as well as $\tilde{k}^{0}$, just cancel, which is that in the genesis phase with $n_{T}=2[20]$. While for $\tilde{k} \gg 1$,

$$
\mathcal{P}_{T} \simeq \mathcal{P}_{T, i n f}
$$


which is that of slow-roll inflation. The result is consistent with the evolutions of the perturbation modes plotted in Fig.1. However, for the mode with $\tilde{k} \sim 1$, i.e. $1 / k \sim 1 / \mathcal{H}_{*}$ is around the comoving cutoff scale, we have $n_{T} \sim 1$. We plot $\mathcal{P}_{T}(k)$ in the inset of Fig.2, in which for $k>\mathcal{H}_{*}$, the spectrum is almost scale-invariant with a decaying oscillation, and for $k<\mathcal{H}_{*}$ it gets a cutoff.

We modify the Boltzmann code CAMB[37] to calculate the lensed BB power spectrum, which is plotted in Fig 3 . We set the comoving cutoff scale $1 / \mathcal{H}_{*}$ at $l \sim 60$, at which $n_{T} \sim 1$, and see that the large-scale cutoff of the primordial tensor spectrum (12) brings a BB power suppression.

Noting that $n_{T} \gtrsim 1$ is conflicted with the observations of the pulsar timing, BBN, LIGO at smaller scales, which put $n_{T}<0.4$ [8], see also [11]. Here, interestingly, we have $n_{T}>1$ at large scale, but $n_{T} \simeq 0$ at small scale, which makes our scenario also comply with the constraints at small scale well.

There is generally a recombination peak at $l \sim 80$ and a reionization bump at $l<10$ in $\Lambda \mathrm{CDM}+r$ power-law model. Both are the imprints of the primordial tensor perturbation. The detection of the recombination peak will be a confirmation that inflation has ever occurred, while Planck with all-sky coverage will highly-probably detect the reionization bump at low-l, which might help us to understand the physics of pre-inflationary universe. The amplitude and shape of the reionization bump record the reionization history of universe. However, since we have $n_{T}=2$ at larger scale, the perturbation modes at low- $l$ will acquire a larger suppression, which makes the reionization bump in our BB power spectrum hardly visible. The upcoming Planck polarization data may falsify our scenario. The effect of $n_{T} \gtrsim 1$ on the reionization bump in $\mathrm{BB}$ power spectrum is estimated in Appendix. In addition, the large reduction of tensor amplitude at large scale also helps to reduce power in TT power spectrum at low-l, where Planck showed a deficit.

Here, we set the comoving cutoff scale $1 / \mathcal{H}_{*}$ at $l \sim 60$. It should be mentioned that if $1 / \mathcal{H}_{*}$ is at $l \sim 10$, the suppression of the reionization bump in BB power spectrum will be weaken, a low bump will appear, like in Ref.[38]. We will study such a case elsewhere.

In summary, we have proposed an inflationary scenario, in which the pre-inflationary evolution of the primordial universe is a slowly expanding genesis phase. Predictably, with this scenario, we will not see the reionization bump in BB power spectrum at $l<10$, which will highly-probably detected by Planck. The pre-inflationary genesis requires the dramatic 
violation of NEC, which might have potential implications, e.g. [39], [40], [41], the upcoming Planck polarization data would tell whether it has ever occurred.

\section{Acknowledgments}

This work was talked in "Advanced Seminar" on Apr.16, organized by Jianxin Lu. We thank Yungui Gong, Qing-Guo Huang, Mingzhe Li for discussions during the seminar. YSP is supported by NSFC, No. 11222546, and National Basic Research Program of China, No.2010CB832804. HL is supported by NSFC, No. 11322325 and 11033005.

\section{Appendix: The parameterization of tensor spectrum with large-scale cutoff}

We will estimate the effect of $n_{T} \gtrsim 1$ on the reionization bump in BB power spectrum at $l<10 . \mathcal{P}_{T}$ may be parameterized as

$$
\mathcal{P}_{T}=\frac{\mathcal{P}_{T, i n f}}{1+A_{*}\left(\frac{\mathcal{H}_{*}}{k}\right)^{n_{\text {cutoff }}}},
$$

where $\mathcal{P}_{T, \text { inf }}$ is that of the slow-roll inflation, both $A_{*}$ and $n_{\text {cutoff }}$ are parameters reflecting the shape of $\mathcal{P}_{T}$. We have $n_{T} \simeq 0$ for $k \gg \mathcal{H}_{*}$ and $n_{T} \simeq n_{\text {cutoff }}$ for $k \ll \mathcal{H}_{*}$. Thus the value of $n_{\text {cutoff }}$ sets a large-scale cutoff.

When $n_{\text {cutoff }}=2$, the spectrum (15) may simulate (12) with a negligible oscillation in the regime $k>\mathcal{H}_{*}$. When $n_{\text {cutoff }}=1$, it is that in Ref.[42], in which the pre-inflationary expanding phase is a superinflationary evolution

$$
a \sim \frac{1}{H_{*}\left(t_{*}-t\right)}
$$

and $\epsilon \simeq-1$. Here, the superinflation is defined as the evolution with $\dot{H} / H^{2}>0$. Thus the slowly expanding genesis also belongs to the superinflation, but since $\epsilon \ll-1$, the expansion is actually slow.

It is validated in Ref.[43] that if the pre-inflationary universe is expanding, $n_{T} \leqslant 2$, and if it is contracting, $n_{T} \leqslant 3$. Thus for $n_{\text {cutoff }}=3$, the pre-inflationary universe should be contracting, it may be that in bounce inflation scenario [26], [27], in which before the slowroll inflation the universe is in a contracting phase with $a \sim\left(t_{*}-t\right)^{1 / 3}$, on which a cyclic universe may be based [44]. Here, the same cutoff also appears in scalar spectrum. However, since the comoving cutoff scale for the scalar spectrum is hardly extended to $l>10$, or the model is not consistent with Planck temperature data, thus the reionization bump of BB 


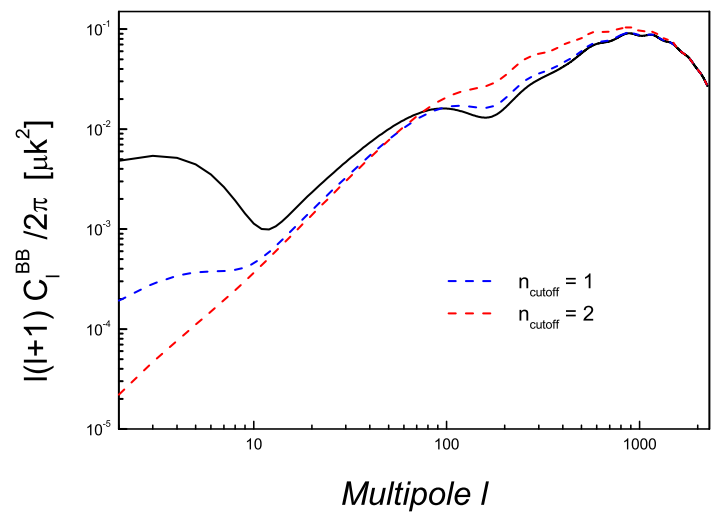

FIG. 4: Theoretical CMB BB power spectra for the conventional slow-roll inflationary scenario (black solid line), in which the tensor spectrum is power-law, and $\Omega_{b} h^{2}=0.02184, \Omega_{c} h^{2}=0.118$, $\tau=0.08,100 \Theta_{s}=1.04, n_{\mathcal{R}}=0.96 A_{s}=2.14 \times 10^{-9}$, and our parameterized model, in which $n_{T}$ is replaced with the parameterization (15). We consider two cases: $n_{\text {cutoff }}=1$ (blue dashed line) and $n_{\text {cutoff }}=2$ (red dashed line).

power spectrum in bounce inflation will still exist but slightly lower [38], see also [45], 46] for other pre-inflationary contractions.

We plot the effect of $n_{\text {cutoff }} \gtrsim 1$ on the reionization bump at $l<10$ in Fig,4. When $n_{\text {cutoff }}=2$, there is not the reionization bump, since the tilt of $\mathcal{P}_{T}$ is large, which leads to a highly large reduction of the perturbation modes at low- $l$ and makes the reionization bump hardly visible. When $n_{\text {cutoff }}=1$, the reionization bump will appear but be compressed compared with that of $n_{\text {cutoff }}=0$. The parameterization (15) actually helps to qualitatively distinguish the inflationary models with different pre-inflationary evolutions giving different $n_{\text {cutoff }}$.

[1] P. A. R. Ade et al. [BICEP2 Collaboration], arXiv:1403.3985 [astro-ph.CO].

[2] R. Adam et al. [Planck Collaboration], arXiv:1409.5738 [astro-ph.CO].

[3] M. J. Mortonson and U. Seljak, arXiv:1405.5857 [astro-ph.CO].

[4] J. Lizarraga, J. Urrestilla, D. Daverio, M. Hindmarsh, M. Kunz and A. R. Liddle, arXiv:1403.4924 [astro-ph.CO]. 
[5] A. Moss and L. Pogosian, arXiv:1403.6105 [astro-ph.CO].

[6] P. A. R. Ade et al. [Planck Collaboration], arXiv:1303.5075 [astro-ph.CO].

[7] H. Li, J. -Q. Xia and X. Zhang, arXiv:1404.0238 [astro-ph.CO].

[8] K. M. Smith, C. Dvorkin, L. Boyle, N. Turok, M. Halpern, G. Hinshaw and B. Gold, Phys. Rev. Lett. 113, 031301 (2014) [arXiv:1404.0373 [astro-ph.CO]].

[9] J. Martin, C. Ringeval, R. Trotta and V. Vennin, arXiv:1405.7272 [astro-ph.CO].

[10] A. Lewis, http://cosmocoffee.info/viewtopic.php?t=2302].

[11] M. Gerbino, A. Marchini, L. Pagano, L. Salvati, E. Di Valentino and A. Melchiorri, Phys. Rev. D 90, 047301 (2014) arXiv:1403.5732 [astro-ph.CO]].

[12] B. Hu, J. W. Hu, Z. K. Guo and R. G. Cai, Phys. Rev. D 90, 023544 (2014) arXiv:1404.3690 [astro-ph.CO]].

[13] Y. -H. Li, J. -F. Zhang, X. Zhang, arXiv:1405.0570 [astro-ph.CO].

[14] S. Mukohyama, R. Namba, M. Peloso and G. Shiu, arXiv:1405.0346 [astro-ph.CO].

[15] Y. f. Cai and Y. S. Piao, Phys. Lett. B 657, 1 (2007) gr-qc/0701114; Y. F. Cai and Y. Wang, Phys. Lett. B 735, 108 (2014) [arXiv:1404.6672 [astro-ph.CO]].

[16] A. Ashoorioon, K. Dimopoulos, M. M. Sheikh-Jabbari and G. Shiu, JCAP 1402, 025 (2014) arXiv:1306.4914 [hep-th]]; Phys. Lett. B 737, 98 (2014) [arXiv:1403.6099 [hep-th]].

[17] Y. Wang and W. Xue, arXiv:1403.5817 [astro-ph.CO].

[18] Y. -S. Piao and Y. -Z. Zhang, Phys. Rev. D 70, 063513 (2004) astro-ph/0401231.

[19] M. Baldi, F. Finelli and S. Matarrese, Phys. Rev. D 72, 083504 (2005) astro-ph/0505552.

[20] P. Creminelli, A. Nicolis and E. Trincherini, JCAP 1011, 021 (2010) arXiv:1007.0027 [hepth]].

[21] Z. -G. Liu, J. Zhang and Y. -S. Piao, Phys. Rev. D 84, 063508 (2011) arXiv:1105.5713 ]; Z.-G. Liu and Y.-S. Piao, Phys. Lett. B 718, 734 (2013) [arXiv:1207.2568].

[22] K. Hinterbichler, A. Joyce, J. Khoury and G. E. J. Miller, JCAP 1212, 030 (2012) arXiv:1209.5742 [hep-th]]; Phys. Rev. Lett. 110, 24, 241303 (2013) arXiv:1212.3607 [hepth]].

[23] Y. S. Piao and E. Zhou, Phys. Rev. D 68, 083515 (2003) hep-th/0308080.

[24] V. A. Rubakov, arXiv:1401.4024 [hep-th].

[25] J. Khoury, B. A. Ovrut, P. J. Steinhardt and N. Turok, Phys. Rev. D 64, 123522 (2001) hep-th/0103239]; J. -L. Lehners and P. J. Steinhardt, Phys. Rev. D 87, no. 12, 123533 (2013) 
arXiv:1304.3122 [astro-ph.CO]].

[26] Y. S. Piao, B. Feng and X. m. Zhang, Phys. Rev. D 69, 103520 (2004) hep-th/0310206;

Y. S. Piao, Phys. Rev. D 71, 087301 (2005) astro-ph/0502343.

[27] Z. G. Liu, Z. K. Guo and Y. S. Piao, Phys. Rev. D 88, 063539 (2013) arXiv:1304.6527.

[28] E. Dudas, N. Kitazawa, S. P. Patil and A. Sagnotti, JCAP 1205 (2012) 012; N. Kitazawa and A. Sagnotti, arXiv:1402.1418 [hep-th].

[29] E. Ramirez, D. J. Schwarz, Phys. Rev. D 85, 103516 (2012) [arXiv:1111.7131 [astro-ph.CO]].

[30] A. Borde, A. H. Guth and A. Vilenkin, Phys. Rev. Lett. 90, 151301 (2003) gr-qc/0110012.

[31] J. Khoury and G. E. J. Miller, Phys. Rev. D 84, 023511 (2011) [arXiv:1012.0846 [hep-th]]; A. Joyce and J. Khoury, Phys. Rev. D 84, 023508 (2011) arXiv:1104.4347 [hep-th]].

[32] G. Geshnizjani, W. H. Kinney and A. M. Dizgah, JCAP 1202, 015 (2012) arXiv:1110.4640 [astro-ph.CO]].

[33] A. Nicolis, R. Rattazzi and E. Trincherini, Phys. Rev. D 79, 064036 (2009) arXiv:0811.2197 [hep-th]].

[34] Y. -S. Piao, Phys. Lett. B 701, 526 (2011) [arXiv:1012.2734].

[35] V. A. Rubakov, JCAP 0909, 030 (2009) [arXiv:0906.3693 [hep-th]].

[36] K. Hinterbichler and J. Khoury, JCAP 1204, 023 (2012) arXiv:1106.1428 [hep-th]].

[37] A. Lewis, A. Challinor and A. Lasenby, Astrophys. J. 538, 473 (2000) astro-ph/9911177];

A. Challinor and A. Lewis, Phys. Rev. D 71, 103010 (2005) astro-ph/0502425.

[38] Y. T. Wang and Y. S. Piao, arXiv:1409.7153 [gr-qc].

[39] V. A. Rubakov, Phys. Rev. D 88, 044015 (2013) [arXiv:1305.2614 [hep-th]].

[40] Z. -G. Liu and Y. -S. Piao, Phys. Rev. D 88, 043520 (2013) arXiv:1301.6833 [gr-qc]].

[41] B. Elder, A. Joyce and J. Khoury, Phys. Rev. D 89, 044027 (2014) [arXiv:1311.5889 [hep-th]].

[42] Z. G. Liu, Z. K. Guo and Y. S. Piao, Eur. Phys. J. C 74, 3006 (2014) arXiv:1311.1599 [astro-ph.CO]].

[43] Y. -S. Piao and Y. -Z. Zhang, Phys. Rev. D 70, 043516 (2004) astro-ph/0403671.

[44] Y. -S. Piao, Phys. Rev. D 70, 101302 (2004) hep-th/0407258; Phys. Lett. B 677, 1 (2009) arXiv:0901.2644 [gr-qc]].

[45] J. Liu, Y. -F. Cai and H. Li, J. Theor. Phys. 1, 1 (2012) arXiv:1009.3372 [astro-ph.CO]]; J. -Q. Xia, Y. -F. Cai, H. Li and X. Zhang, arXiv:1403.7623 [astro-ph.CO].

[46] T. Qiu, arXiv:1404.3060 [gr-qc]. 